\title{
Pulmonary reperfusion injury after the unifocalization procedure for tetralogy of Fallot, pulmonary atresia, and major aortopulmonary collateral arteries
}

\author{
Shiraz A. Maskatia, MD, ${ }^{\text {a }}$ Jeffrey A. Feinstein, MD, MPH, ${ }^{b}$ Beverley Newman, MD, ${ }^{c}$ \\ Frank L. Hanley, MD, ${ }^{\mathrm{d}}$ and Stephen J. Roth, MD, MPH ${ }^{\mathrm{b}}$
}

\begin{abstract}
Objective: The aims of our study are to describe the incidence, clinical profile, and risk factors for pulmonary reperfusion injury after the unifocalization procedure for tetralogy of Fallot, pulmonary atresia, and major aortopulmonary collateral arteries. We hypothesized the following: (1) Pulmonary reperfusion injury is more likely to occur after unifocalization procedures in which a septated circulation is not achieved, (2) pulmonary reperfusion injury is directly related to the severity of stenosis in major aortopulmonary collateral arteries, and (3) pulmonary reperfusion injury leads to longer intubation time and longer hospitalization.
\end{abstract}

\begin{abstract}
Methods: Consecutive patients with tetralogy of Fallot/pulmonary atresia/major aortopulmonary collateral arteries who underwent unifocalization procedures over a 5-year period were identified in our institutional database. Chest radiographs before the unifocalization procedure, from postoperative days 0 to 4 , and from 2 weeks after the unifocalization procedure or at discharge were evaluated by a pediatric radiologist for localized pulmonary edema. Determination of stenosis severity was based on review of preoperative angiograms. Statistical analyses using multivariate repeated-measures analyses were performed with generalized estimating equations.
\end{abstract}

Results: Pulmonary reperfusion injury was present after 42 of 65 (65\%) unifocalization procedures. In 36 of 42 cases of reperfusion injury, unilateral injury was present. Risk factors for the development of reperfusion injury included bilateral unifocalization $(P=.01)$ and degree of stenosis $(P=.03)$. We did not identify an association between pulmonary reperfusion injury and time to tracheal extubation or hospital discharge.

Conclusions: Pulmonary reperfusion injury is common after the unifocalization procedure for tetralogy of Fallot/pulmonary atresia/major aortopulmonary collateral arteries. Severity of stenosis and bilateral unifocalization are associated with the development of reperfusion injury. (J Thorac Cardiovasc Surg 2012;144:184-9)

Earn CME credits at

http://cme.ctsnetjournals.org

Tetralogy of Fallot (TOF) with pulmonary atresia (PA) is a heterogeneous form of congenital heart disease that can be classified by the source of pulmonary blood flow. Pulmonary blood flow can be supplied exclusively by intrapericardial pulmonary arteries fed by a patent ductus arteriosus, by both intrapericardial pulmonary arteries and major aortopulmonary collateral arteries (MAPCAs), or solely by MAPCAs. ${ }^{1,2}$ Patients with diminutive central pulmonary

From Division of Pediatric Cardiology, 'Texas Children's Hospital, Baylor College of Medicine, Houston, Tex; and Division of Pediatric Cardiology, ${ }^{b}$ Department of Radiology, ${ }^{\mathrm{c}}$ and Department of Cardiothoracic Surgery, ${ }^{\mathrm{d}}$ Stanford University Medical Center, Palo Alto, Calif.

Received for publication June 19, 2011; revisions received Sept 12, 2011; accepted for publication Dec 14, 2011; available ahead of print Jan 13, 2012.

Address for reprints: Shiraz A. Maskatia, MD, Baylor College of Medicine, Pediatric Cardiology, 6621 Fannin, West Tower, Houston, TX 77030 (E-mail: samaskat@ texaschildrenshospital.org)

0022-5223/\$36.00

Copyright (C) 2012 by The American Association for Thoracic Surgery doi:10.1016/j.jtcvs.2011.12.030 arteries and MAPCA-dependent pulmonary blood flow represent a higher-risk category of patients. ${ }^{3,4}$ To achieve a septated circulation, patients with MAPCAs accounting for a significant proportion of pulmonary blood flow must undergo a unifocalization procedure, which incorporates the MAPCAs into the true pulmonary arteries. This can occur in a single-stage procedure, with closure of the ventricular septal defect (VSD) and septation of the systemic and pulmonary circulations, or stepwise procedures with initial unifocalization of the MAPCAs to a systemic-to-pulmonary artery shunt followed later by complete repair., ${ }^{3,5-7}$

Pulmonary reperfusion injury in the setting of pulmonary artery angioplasty for pulmonary artery stenosis or chronic thromboembolic disease is a well-recognized clinical entity. ${ }^{8-10}$ It typically manifests by temporary, localized pulmonary edema in an area of increased perfusion of lung tissue that impairs gas exchange. It has been hypothesized that this injury is due to increased blood flow in areas of previously underperfused lung. Pulmonary injury after cardiopulmonary bypass has been shown to lead to prolonged hospitalization, longer intubation time, and increased hospitalization cost. ${ }^{11}$ To our knowledge, pulmonary reperfusion injury after the unifocalization procedure has not been described. The aims of 

Abbreviations and Acronyms
GEE = generalized estimating equation
MAPCA $=$ major aortopulmonary collateral artery
PA = pulmonary atresia
TOF $=$ tetralogy of Fallot
VSD $=$ ventricular septal defect

our study are to describe the incidence, clinical profile, and risk factors for the development of pulmonary reperfusion injury after the unifocalization procedure for TOF/PA/ MAPCAs. Cases in which a septated circulation is not achieved would be expected to result in greater blood flow to distal lung tissue compared with cases in which the VSD is closed. In addition, cases with significant preoperative MAPCA stenosis would be expected to have a higher increase in flow to distal lung tissue compared with those with lower degrees of stenosis. Therefore, we hypothesized that pulmonary reperfusion injury is more likely to be present after unifocalization procedures in which a septated circulation is not achieved and in those with more severe MAPCA stenosis. Furthermore, we hypothesized that pulmonary reperfusion injury leads to longer postoperative tracheal intubation time and length of hospitalization.

\section{MATERIALS AND METHODS}

We performed a retrospective review of our surgical database for patients with TOF/PA/MAPCAs who received a unifocalization procedure from November 30, 2001, to July 20, 2006. During the study period, we used a standardized surgical strategy to patients undergoing unifocalizations. ${ }^{12,13}$ Briefly, this strategy involved performing a unifocalization plus a complete repair with VSD closure via median sternotomy when the pulmonary arteries could be adequately reconstructed. If VSD closure was deferred at the time of unifocalization, an appropriately sized central aortopulmonary shunt was inserted. This study was performed with approval of the Stanford University Medical Center Institutional Review Board. We included only those with MAPCAs as their sole source of pulmonary blood supply. Patients who developed clinical signs of a postoperative pulmonary hemorrhage (identified in the medical record as having frankly bloody secretions from the endotracheal tube along with a significant decrease in the hematocrit and a pulmonary opacity on chest radiograph) or pneumonia (identified in the medical record as developing an elevated white blood cell count, left shift of the white blood cell count, fever, endotracheal tube aspirate with elevated polymorphonuclear lymphocytes, and a pulmonary opacity on chest radiograph) were excluded from the study.

One anterior-posterior chest radiograph was obtained for each of the following time frames: preoperative, postoperative day 0 to 4 , and 2 weeks postoperative or at discharge from the hospital (if discharge from the hospital was before 2 weeks after the operation). In total, 7 chest radiographs were obtained for each unifocalization procedure performed. Characteristic preoperative, postoperative day 2 , and discharge radiographs of a patient with reperfusion injury are provided (Figure 1). Each radiograph was scored by 1 faculty pediatric radiologist who was blinded to the identity, clinical course, and operative variables of the patients. Only the age of the patient was provided. The radiographs were scored using a pulmonary edema scoring system adapted from a previous study assessing pulmonary reperfusion injury in lung transplant recipients. ${ }^{14}$ The right upper lobe, right middle lobe, right lower lobe, left upper lobe, lingual, and left lower lobe were all scored on a scale from 0 to 3 . The degree of edema in each lobe was assessed by using a 4-point scale: 0 for normal lung, 1 for minimal opacity not obscuring lung vessels, 2 for opacity partially obscuring lung vessels, and 3 for opacity completely obscuring lung vessels. Lung vascularity and the presence or absence of atelectasis was also recorded. In cases of oligemic or hyperemic lung fields, the edema was scored relative to normal pulmonary vascularity. Otherwise, the lung vascularity assignment did not affect the opacity score. The suitability of the film for inclusion in the study was at the discretion of the radiologist.

For the purposes of this study, pulmonary reperfusion injury was defined as a change in pulmonary opacity appearing after the unifocalization procedure that resolved within 2 weeks or before discharge and was not associated with pulmonary hemorrhage, pneumonia, or atelectasis. Reperfusion injury was analyzed as both a binary and an ordinal outcome. When analyzed as a binary outcome, a change in edema score of 2 or more was used to define reperfusion injury.

The level of stenosis of each MAPCA was obtained from angiograms from catheterization studies performed before each unifocalization procedure. All measurements were performed by a pediatric resident. One third of MAPCAs also were randomly chosen to be measured by a pediatric interventional cardiologist for comparison. The width of the narrowest segment of each MAPCA in millimeters was measured. The widest downstream segment was then measured. If a MAPCA branched into multiple segments before the largest downstream segment, the total width of all downstream segments was used. The stenosis was then reported as a ratio of the width of the narrowest segment to the width of the largest segment downstream (or total width of all branch segments). When analyzing the stenosis ratio as a risk factor for reperfusion injury, time to extubation, and time to hospital discharge, the smallest stenosis ratio for each lung was used. When 2 stenoses were present distal to a branch point, each stenosis was measured independently, and the smaller stenosis was used.

Laboratory data were also obtained from the medical record over the study period. The highest white blood cell count, highest serum C-reactive protein, lowest hematocrit, and lowest serum albumin were recorded. These data were obtained from the time of surgery to discharge or 2 weeks after surgery.

\section{Data Analysis}

Patients were analyzed for degree of change in pulmonary opacity from the preoperative radiograph to the maximum degree of opacity in the first 4 postoperative days. To exclude patients with other sources of pulmonary opacity, patients with a preoperative radiograph with an opacity score greater than 1 were excluded from the reperfusion injury analysis. In addition, because pulmonary reperfusion injury is thought to be a self-limited phenomenon, patients who had discharge/2-week radiographs that reflected opacity greater than 1 were also excluded from the reperfusion injury analysis. We analyzed the degree of change in opacity score in reference to a number of risk factors. These risk factors included the number of unifocalization procedures performed, age at surgery, degree of stenosis of unifocalized segment on preoperative catheterization, laterality of repair (unilateral vs bilateral unifocalization), use of cardiopulmonary bypass, type of repair (full intracardiac repair with right ventricle-topulmonary artery conduit vs staged procedure with placement of a systemic-to-pulmonary artery shunt while leaving the VSD open), number of vessels unifocalized, lowest serum albumin, lowest serum hematocrit, highest white blood cell count, length of time tracheally intubated, and length of postoperative stay in the intensive care unit.

Data collection and descriptive statistics were performed using Microsoft Excel (Microsoft Corp, Redmond, Wash). We used generalized estimating equations (GEEs) using the GENMOD procedure. GEEs are used to model correlated data from multilevel studies in which one would otherwise use generalized linear models. ${ }^{15}$ They take into account the 


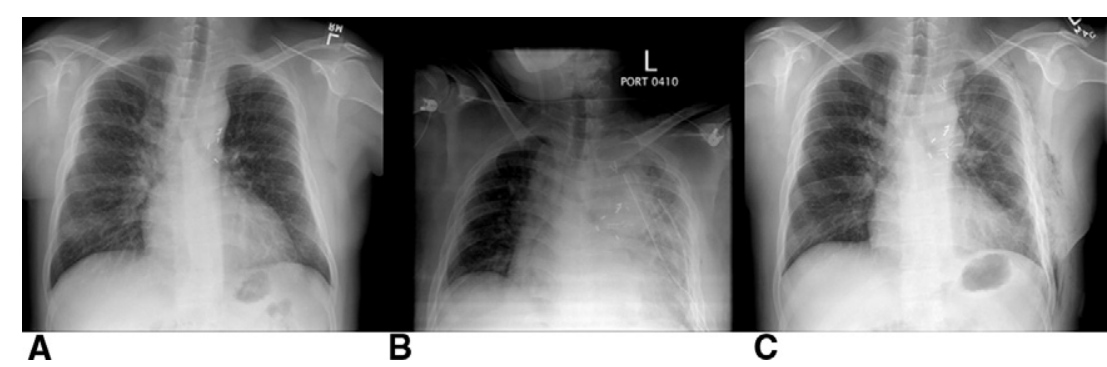

FIGURE 1. Characteristic series of chest radiographs of a patient after unifocalization with left-sided pulmonary reperfusion injury. Preoperative (A), postoperative day 2 (B), and discharge chest radiograph (C).

covariance due to multiple observations from a single subject. This approach was required because many patients received multiple unifocalization procedures during the course of the study. Correlation between stenosis ratio measurements obtained by the pediatrics resident and the pediatric interventional cardiologist was assessed with Pearson's productmoment correlation. In the case of unilateral unifocalization procedures, a Wilcoxon sign-rank test was performed to assess the degree of edema in the lung operated on compared with the contralateral lung. All statistical analyses were performed using SAS 9.22 (SAS Institute Inc, Cary, NC) by a biostatistician.

\section{RESULTS}

Between November 30, 2001, and July 7, 2006, 59 patients with TOF/PA/MAPCAs underwent 92 unifocalization procedures. This was the initial cardiac surgery in 51 of $92(55 \%)$ unifocalization procedures and was a subsequent surgery in 41 of $92(45 \%)$. The unifocalization procedure was unilateral in 52 of $92(56 \%)$. A total of 17 unifocalization procedures were excluded from the study. Table 1 depicts the reasons for exclusion from the study.

Table 2 depicts the baseline characteristics of patients included in the study. A total of 65 unifocalization procedures remained and were included in the analysis. Of these, reperfusion injury was present in $42(65 \%)$, of which $36(86 \%)$ were unilateral. In cases of unilateral reperfusion injury, we performed a Wilcoxon sign-rank analysis to determine whether the side unifocalized was the side that exhibited reperfusion injury. The change in opacity score on the side operated on was consistently higher than the change in opacity score on the side not operated on $(P<.01)$.

In 13 of these 65 unifocalization procedures, angiographic data were inadequate to determine the degree of

TABLE 1. Operations excluded

\begin{tabular}{ll}
\hline \multicolumn{1}{c}{ Operations excluded } & $\mathbf{N = \mathbf { 2 7 }}$ \\
\hline Urgent reoperation & $2(7 \%)$ \\
Death within 2 wk postoperatively & $2(7 \%)$ \\
Pneumonia & $6(22 \%)$ \\
Pulmonary hemorrhage & $3(11 \%)$ \\
Insufficient radiographs & $4(15 \%)$ \\
Baseline opacity score $>1$ & $1(4 \%)$ \\
Discharge/2-wk opacity score $>1$ & $6(22 \%)$ \\
Radiograph quality otherwise insufficient & $3(11 \%)$ \\
\hline
\end{tabular}

MAPCA stenosis. The remaining 52 unifocalization procedures were included in the multivariate analysis for reperfusion injury. In 30 of 52 cases (58\%), patients demonstrated radiographic evidence of reperfusion injury.

\section{Risk Factors for Reperfusion Injury}

Operative data. When reperfusion injury was analyzed as a binary outcome, bilateral unifocalization correlated with an increased risk of reperfusion injury $(P=.01$, Table 3$)$. When analyzed as an ordinal outcome, this correlation was not statistically significant. In 16 of $92(17 \%)$ unifocalization procedures, the VSD was closed. VSD closure (creating a septated circulation), the use of cardiopulmonary bypass, and the number of vessels unifocalized did not correlate with the development of reperfusion injury. Stenosis ratio. The minimum stenosis ratio was 0.126 , and the maximum ratio was 0.831 . The mean stenosis ratio \pm standard deviation was $0.377 \pm 0.166$. The GEE procedure demonstrated that a smaller stenosis ratio correlated with an increased risk of pulmonary reperfusion injury when analyzed as a binary outcome $(P=.03$, Table 3$)$. When reperfusion injury was analyzed as an ordinal outcome, similar results were found. The level of agreement between the pediatric resident and the interventional cardiologist was excellent $(r=0.85)$.

Reperfusion injury was present in 18 of 31 (58\%) bilateral unifocalization procedures. In 13 of these 18 cases, the injury was unilateral. The mean stenosis ratio in the affected side was $0.27 \pm 0.09$ compared with $0.51 \pm 0.11$ in the unaffected side $(P=.03)$.

Age at unifocalization. The median age of patients undergoing unifocalization included in the study was 1.9 years (range, 0.19-27 years). The median age at unifocalization of the group who exhibited reperfusion injury was 1.4 years (range, 0.3-27 years), and the median age at surgery of the group who did not exhibit reperfusion injury was 2.2 years (range, $0.3-25$ years). Although GEE analysis indicated that there was a statistically significant correlation between younger age at unifocalization and the development of reperfusion injury $(P=.04)$, the odds ratio was 0.99 , indicating a small effect size (Table 3). 
TABLE 2. Baseline data

\begin{tabular}{lc}
\hline \multicolumn{1}{c}{ No. of patients } & $\mathbf{N}=\mathbf{4 4}$ \\
\hline Male (\%) & $25(57 \%)$ \\
No. of operations & 65 \\
Median (range) age at time of unifocalization (y) & $1.9(0.19-27)$ \\
Unilateral unifocalizations (no. of operations) & $34(52 \%)$ \\
Bilateral unifocalizations (no. of operations) & $31(48 \%)$ \\
VSD closure (no. of operations) & $16(25 \%)$ \\
CPB (no. of operations) & $40(62 \%)$ \\
Median (range) CPB time (min) & $162(0-242)$ \\
\hline
\end{tabular}

$V S D$, Ventricular septal defect; $C P B$, cardiopulmonary bypass.

Laboratory data. The highest white blood cell count, highest serum C-reactive protein, and lowest serum albumin did not correlate with reperfusion injury. The lowest serum hematocrit ranged from $32.3 \%$ to $54.1 \%$, with a mean \pm standard deviation of $41.6 \% \pm 6.1 \%$. In cases in which reperfusion injury was not evident, the lowest hematocrit was an average of $39.6 \% \pm 5.7 \%$. In cases in which reperfusion injury was evident, the lowest hematocrit was an average of $42.8 \% \pm 6.0 \%$. Although by GEE analysis there was a significant correlation between the lowest serum hematocrit and the development of reperfusion injury, the odds ratio was 1.1 , indicating a small effect size (Table 3).

\section{Risk Factors for Time to Tracheal Extubation}

The median time to extubation was 4 days (range, 1-67 days). The median time to extubation in patients without reperfusion injury was 3 days (range, 1-9 days), whereas it was 4 days (range, 2-67 days) in those with reperfusion injury. In the case with an intubation time of 67 days, the postoperative course was complicated by Enterococcal endocarditis, which was treated medically. Analysis by GEE demonstrated no significant association between reperfusion injury and time intubated. VSD closure, age, number of vessels unifocalized, and bilateral repair also did not correlate with time to extubation.

\section{Risk Factors for Length of Hospital Stay}

The median length of hospital stay was 13 days (range, 5-98 days). The median time to discharge in patients without reperfusion injury was 10 days (range, 533 days), whereas it was 13 days (range, 5-98 days) in those with reperfusion injury. The case with a hospitalization of 98 days was the same case as the aforementioned patient with a postoperative course complicated by Enterococcal endocarditis. Analysis with GEE demonstrated no significant association between the diagnosis of reperfusion injury and the length of hospital stay. VSD closure, number of vessels unifocalized, age at surgery, and bilateral repair also did not correlate with length of hospital stay.
TABLE 3. Risk factors for reperfusion injury

\begin{tabular}{llcc}
\hline & OR & $\mathbf{9 5 \%}$ CI & $\boldsymbol{P}$ value \\
\hline Bilateral repair & 6.7 & $1.7-27.1$ & .01 \\
No. of vessels & 1.0 & $0.6-1.57$ & .999 \\
Stenosis ratio & 0.0005 & $0.0000006-0.42$ & .03 \\
Age at unifocalization (y) & 0.99 & $0.98-0.99$ & .04 \\
White blood count & 0.92 & $0.84-1.0$ & .10 \\
Hematocrit & 1.1 & $1.1-1.1$ & .03 \\
Serum albumin & 0.49 & $0.23-1.0$ & .07 \\
\hline
\end{tabular}

$O R$, Odds ratio; $C I$, confidence interval.

\section{DISCUSSION}

We performed a retrospective review of patients who underwent the unifocalization procedure for TOF/PA/MAPCAs to determine the incidence of and risk factors associated with pulmonary reperfusion injury. We found that pulmonary reperfusion injury was present after 42 of $65(65 \%)$ unifocalization procedures. In 36 of $42(86 \%)$ cases of reperfusion injury, unilateral injury was present. Risk factors for the development of reperfusion injury included bilateral unifocalization and degree of stenosis. We did not identify an association between pulmonary reperfusion injury and time to tracheal extubation or hospital discharge.

This is the first study to describe postoperative pulmonary reperfusion injury after the unifocalization procedure for TOF/PA/MAPCAs. We believe this injury is distinct from pulmonary injury seen after cardiopulmonary bypass. Postoperative pulmonary injury after cardiopulmonary bypass is a well-described entity that can result in prolonged hospital stay, prolonged intubation, and increased hospital costs. ${ }^{11}$ Clinical studies in humans have not found differences in rates of pulmonary injury postbypass comparing deep hypothermic low-flow bypass and deep hypothermic circulatory arrest. ${ }^{11}$ However, a recent animal study showed an increase in pulmonary tissue inflammatory markers after deep hypothermic circulatory arrest compared with hypothermia alone and low-flow cardiopulmonary bypass. ${ }^{16}$ The investigators hypothesized that this difference was due to pulmonary ischemia-reperfusion injury. Although there may be similar physiologic mechanisms at play, these studies focus on clinical scenarios that are different from those occurring in the patients we studied. In our study, the use of cardiopulmonary bypass did not correlate with increased risk of developing reperfusion injury. However, bilateral surgery did correlate significantly with risk of developing pulmonary reperfusion injury. Longer operative times may lead to increased risk of clinically significant pulmonary reperfusion injury. Our study suggests that this is not a reflection of the effect of cardiopulmonary bypass, but may be due to other operative factors. The extensive hilar dissection typically involved in these procedures may lead to the mechanical disruption of the innervation of the 
pulmonary vasculature and disruption of hilar lymphatic vessels.

We found that $65 \%$ of patients who underwent unifocalization experienced radiographic evidence of reperfusion injury. Despite the high index of suspicion for pulmonary reperfusion injury, the incidence we detected is significantly higher than we expected. Previous reports of pulmonary reperfusion injury in patients after branch pulmonary artery dilation describe incidences of $15 \%$ to $17 \% .^{9,17}$ There are several possible reasons for this difference. First, the postoperative course of patients status after unifocalization is variable. Coincident postoperative derangements of pulmonary mechanics are common, and pulmonary reperfusion injury is more likely to be recognized in a prolonged, difficult postoperative course. In addition, patients may have had other postoperative complications that interfere with the recognition of pulmonary reperfusion injury. Thoracic pathology (pulmonary hemorrhage, pneumonia, or pleural effusion) that obscured the diagnosis of pulmonary reperfusion injury was evident in 19 patients.

We hypothesized that patients with a more "tight" stenosis within a MAPCA to be unifocalized would be at increased risk of pulmonary reperfusion injury. Indeed, a smaller stenosis ratio did correlate with risk of developing pulmonary reperfusion injury when analyzed both as a binary outcome and as an ordinal outcome. This finding has not been published previously. In addition, in patients who underwent bilateral unifocalization and had unilateral reperfusion injury, the mean stenosis ratio was lower in the affected side than the unaffected side. Patients with unrepaired TOF or isolated branch pulmonary artery stenosis who have undergone balloon angioplasty of stenotic pulmonary arteries are at risk of developing postprocedure pulmonary edema. ${ }^{8,9,17}$ This generally occurs within the first 24 hours after the procedure and may lead to longer postprocedure intubation times. Balloon angioplasty of the pulmonary arteries is also used to treat patients with chronic thromboembolic disease and pulmonary hypertension. A retrospective study of 18 patients who underwent 47 catheterization procedures found 11 instances of procedure-related pulmonary edema. ${ }^{10} \mathrm{Pul}-$ monary reperfusion injury during the procedure developed in 4 of the 11 patients and in 7 patients in the subsequent 48 hours. The risk of developing pulmonary reperfusion injury was correlated with a mean pulmonary artery pressure greater than $35 \mathrm{~mm} \mathrm{Hg}$, but did not correlate with age, cardiac index, or size of the dilated pulmonary arterial segment. In regard to the current study, pulmonary artery pressures were not routinely recorded after unifocalization. Therefore, we could not specifically analyze pressure data. We did not detect an association with staged repair (in which the VSD is left open and the patient likely has systemic pulmonary arterial pressure) and the development of reperfusion injury. An acute increase in pulmonary blood flow to areas of lung with vessels that are accustomed to low flow and low pressure may be a main contributor to the development of pulmonary reperfusion injury.

The clinical implications of pulmonary reperfusion injury are difficult to quantify. We did not demonstrate an association between reperfusion injury and length of hospitalization or time to extubation. This is in contrast with pulmonary injury seen after cardiopulmonary bypass. ${ }^{11}$ Although the potential for a type 2 error exists, it is our impression that pulmonary reperfusion injury is common in this context, and that it generates few clinical sequelae. Those caring for patients after the unifocalization procedure should be aware of this condition and its seemingly benign course.

\section{Limitations}

Our study has several limitations. The study design is retrospective and is thus subject to the limitations of this design. After excluding patients with known pneumonia or pulmonary hemorrhage, we based the diagnosis of reperfusion injury on chest radiograph findings. Because we did not measure molecular markers for reperfusion injury, other pathology may have caused the findings we detected. We made every effort to be precise when diagnosing reperfusion injury, and each radiograph was scored independently by a blinded faculty pediatric radiologist. In our effort to be precise concerning the diagnosis of reperfusion injury, we excluded patients who may have had other causes of a pulmonary opacity. As such, a significant number of patients who underwent unifocalization were excluded from the study, which may result in an ascertainment bias. Within the study population, the physical trauma of lung retraction may have led to overlying radiographic opacity and further complicated the pulmonary opacity scoring system. Although our scoring system was amended from a previous study, ${ }^{14}$ it has not been validated. We could not use the International Society of Heart and Lung Transplantation scoring system for reperfusion injury, because this system involves the alveolar oxygen tension:inspired oxygen fraction ratio. ${ }^{18}$ Such a system would not be reliable in many patients in our series, because the source of their pulmonary blood flow was a systemic-topulmonary arterial shunt, and their alveolar oxygen tension was abnormally low because of intracardiac right-to-left shunting. The small number of patients in the reperfusion injury analysis may also result in a type 2 error. This is especially true when analyzing operative variables (VSD closure, the use of cardiopulmonary bypass, and the number of vessels unifocalized). Finally, we only analyzed a limited number of factors that may have contributed to reperfusion injury. There may be other operative, medical, or hemodynamic factors that may contribute to the development of this injury. 


\section{CONCLUSIONS}

Pulmonary reperfusion injury is common in the setting of unifocalization surgery for TOF/PA/MAPCAs, with an incidence of $65 \%$ in our cohort. Factors associated with reperfusion injury included degree of MAPCA stenosis and bilateral unifocalization. We did not demonstrate a significant association between pulmonary reperfusion injury and duration of postoperative tracheal intubation or length of hospitalization. The cause of pulmonary reperfusion injury in the setting of patients who have undergone unifocalization is likely multifactorial. Increased blood flow and pressure to areas of pulmonary vasculature that are exposed to a low-flow state, postbypass pulmonary endothelial dysfunction, ischemia-reperfusion injury, and edema due to mechanical disruption of hilar lymphatic vessels are all possible contributing factors. Further study of this injury is needed. Moving forward, measuring biomarkers of acute lung injury in patients who have clinical evidence of pulmonary reperfusion injury may help to elucidate the mechanism(s) of this injury.

\section{References}

1. Rao BN, Anderson RC, Edwards JE. Anatomic variations in the tetralogy of Fallot. Am Heart J. 1971;81:361-71.

2. Liao PK, Edwards WD, Julsrud PR, Puga FJ, Danielson GK, Feldt RH. Pulmonary blood supply in patients with pulmonary atresia and ventricular septal defect. J Am Coll Cardiol. 1985;6:1343-50.

3. Farouk A, Zahka K, Siwik E, Erenberg F, Al-Khatib Y, Golden A, et al. Individualized approach to the surgical treatment of tetralogy of Fallot with pulmonary atresia. Cardiol Young. 2009;19:76-85.

4. Cho JM, Puga FJ, Danielson GK, Dearani JA, Mair DD, Hagler DJ, et al. Early and long-term results of the surgical treatment of tetralogy of Fallot with pulmonary atresia, with or without major aortopulmonary collateral arteries. $J$ Thorac Cardiovasc Surg. 2002;124:70-81.

5. Reddy VM, McElhinney DB, Amin Z, Moore P, Parry AJ, Teitel DF, et al. Early and intermediate outcomes after repair of pulmonary atresia with ventricular septal defect and major aortopulmonary collateral arteries: experience with 85 patients. Circulation. 2000;101:1826-32

6. Rodefeld MD, Reddy VM, Thompson LD, Suleman S, Moore PC, Teitel DF, et al. Surgical creation of aortopulmonary window in selected patients with pulmonary atresia with poorly developed aortopulmonary collaterals and hypoplastic pulmonary arteries. J Thorac Cardiovasc Surg. 2002;123:1147-54.

7. Duncan BW, Mee RB, Prieto LR, Rosenthal GL, Mesia CI, Qureshi A, et al. Staged repair of tetralogy of Fallot with pulmonary atresia and major aortopulmonary collateral arteries. J Thorac Cardiovasc Surg. 2003;126: 694-702.

8. Kreutzer J, Perry SB, Jonas RA, Mayer JE, Castaneda AR, Lock JE. Tetralogy of Fallot with diminutive pulmonary arteries: preoperative pulmonary valve dilation and transcatheter rehabilitation of pulmonary arteries. J Am Coll Cardiol. 1996;27:1741-7.

9. Bergersen LJ, Perry SB, Lock JE. Effect of cutting balloon angioplasty on resistant pulmonary artery stenosis. Am J Cardiol. 2003;91:185-9.

10. Feinstein JA, Goldhaber SZ, Lock JE, Ferndandes SM, Landzberg MJ. Balloon pulmonary angioplasty for treatment of chronic thromboembolic pulmonary hypertension. Circulation. 2001;103:10-3.

11. Su ZK, Sun Y, Yang YM, Zhang HB, Xu ZW. Lung function after deep hypothermic cardiopulmonary bypass in infants. Asian Cardiovasc Thorac Ann. 2003;11: 328-31.

12. Malhotra SP, Hanley FL. Surgical management of pulmonary atresia with ventricular septal defect and major aortopulmonary collaterals: a protocol-based approach. Semin Thorac Cardiovasc Surg Pediatr Card Surg Annu. 2009;145-51.

13. Reddy VM, Petrossian E, McElhinney DB, Moore P, Teitel DF, Hanley FL. Onestage complete unifocalization in infants: when should the ventricular septal defect be closed? J Thorac Cardiovasc Surg. 1997;113:858-68.

14. Anderson DC, Glazer HS, Semenkovich JW, Pilgram TK, Trulock EP, Cooper JD, et al. Lung transplant edema: chest radiography after lung transplantation-the first 10 days. Radiology. 1995;195:275-81.

15. Zeger SL, Liang KY. Longitudinal data analysis for discrete and continuous outcomes. Biometrics. 1986;42:121-30.

16. Zheng JH, Gao BT, Jiang ZM, Yu XQ, Xu ZW. Evaluation of early macrophage activation and NF-kappaB activity in pulmonary injury caused by deep hypothermia circulatory arrest: an experimental study. Pediatr Cardiol. 2010;31:215-21.

17. Geggel RL, Gauvreau K, Lock JE. Balloon dilation angioplasty of peripheral pulmonary stenosis associated with Williams syndrome. Circulation. 2001;103: 2165-70.

18. Christie JD, Carby M, Bag R, Corris P, Hertz M, Weill D. Report of the ISHLT Working Group on Primary Lung Graft Dysfunction part II: definition. A consensus statement of the International Society for Heart and Lung Transplantation. J Heart Lung Transplant. 2005;24:1454-9. 